\title{
Research Paper \\ Relationship Between Activities of Daily Living and Depression in Older Adults
}

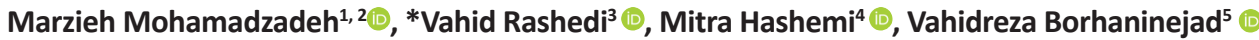

1. Mane and Semelghan Health Center, North Khorasan University of Medical Science, Bojnurd, Iran.

2. PhD Student in Gerontology Iranian Research Center on Aging, University of social Welfare and Rehabilitation Sciences, Tehran, Iran.

3. Department of Gerontology, School of Behavioral Sciences and Mental Health (Tehran Institute of Psychiatry), Iran University of Medical Sciences, Tehran, Iran.

4. Deputy of Research and Technology, North Khorasan University of Medical Sciences, Bojnurd, Iran.

5. Social Determinants of Health Research Center, Institute for Futures Studies in Health, Kerman University of Medical Sciences, Kerman, Iran.

Key words:

Older adults, Depres-

sion, Activities of

daily living

\section{A B S T R A C T}

Objectives Physical disability to perform daily living activities is one of the major causes of depression in the elderly people. On the other hand, depression is associated with loss of interest in daily activities and lack of independence. The present study aimed to assess the relationship of depression with the ability to perform Activities of Daily Living (ADL) and Instrumental Activities of Daily Living (IADL) in older adults in Iran. Methods \& Materials This cross-sectional study was performed on 148 elderly people aged $\geq 60$ years who were selected by multi-stage cluster sampling method. Data were collected by using ADL, IADL, and 15item Geriatric Depression Scale (GDS-15) questionnaires and were then presented by using descriptive statistics and analyzed by Chi-square test, Pearson correlation coefficient, and regression analysis.

Results Participants were 86 female (58.1\%) and 62 male (41.9\%); 109 (73.6\%) aged 60-75 years, and the rest were older than 75 years; 71 (48\%) had moderate or severe depression; 4(2.7\%) and 61(41.2\%) were dependent or in need of help performing ADL and IADL, respectively. Statistical analysis showed that depression has a significant negative correlation with ADL $(r=-0.304, P<0.001)$ and IADL $(r=-0.193, P>0.01)$; i.e. by increasing one of them, the depression decreases.

Conclusion The ability of the elderly people to perform ADL and IADL can be a good predictor of depression in them. With early assessment of their health and ability by health teams and family members, it is possible to prevent the development of their disability and depression.

\section{Extended Abstract}

\section{Introduction}

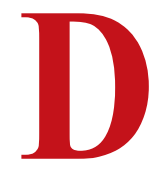

epression is a disorder that is associated with feelings of sadness and hopelessness, and can be considered the most common and important cause of damage to the psychosocial functioning of the elderly $[1,2]$.

Depression often occurs in $12-16 \%$ of the elderly [3] and studies have shown that the prevalence of depression in the Iranian elderly is about 45\% [4]. One of the most important issues in promoting the health and quality of life of the elderly is maintaining their independence in physical and cognitive activities and continuing to live actively [5]. The ability to perform activities reflects an important aspect of functional independence in the elderly [6] and is recognized as an effective factor in maintaining their health and success [7]. One of the important risk factors for depression in the elderly is their physical disability to perform

\section{* Corresponding Author:}

Vahid Rashedi, PhD.

Address: Department of Gerontology, School of Behavioral Sciences and Mental Health (Tehran Institute of Psychiatry), Iran University of Medical Sciences, Tehran, Iran.

Tel: +89 (918) 3071851

E-mail:vahidrashedi@yahoo.com; rashedi.v@iums.ac.ir 
their normal activities, and on the other hand, depression is associated with consequences such as loss of interest in daily activities, increased attachment, and lack of independence [2]. This study aimed to determine the relationship between the ability to perform daily living activities and the rate of depression in the elderly.

\section{Methods \& Materials}

This is a descriptive analytical study with a cross-sectional design. Study population consists of all older people aged $>60$ years living in Maneh \& Samolghan county in Iran. Multi-stage cluster sampling of health centers and then a convenience sampling was used for selecting participants. Inclusion criteria were age over 60 years, residence in the county, and willingness to participate in the study. Those who could not speak and were not mentally aware of the place and time were excluded from the study. The sample size, according to the $\mathrm{r}$ value obtained from 70 pilot samples $(r=0.244)$ and statistical formulas with $95 \%$ confidence level and $80 \%$ test power, was obtained 129 which was determined to be 148 for more confidence.

In order to collect information, a demographic form, Activities of Daily Living (ADL) questionnaire with 7 items[8-10], Lawton Instrumental Activities of Daily Living (IADL) questionnaire [11-13], and 15-item Geriatric Depression Scale (GDS-15) [14] were used. This study has an ethical approval obtained from the Research Ethics Committee of North Khorasan University of Medical Sciences (Code:957/ب/95). Informed consent was obtained from all participants after ensuring them of the confidentiality of their information. Collected data were analyzed in SPSS V. 16 software and were presented by using descriptive statistics (mean and standard deviation). Kolmogorov-Smirnov test was used to evaluate the normality of quantitative data, and Chi-square test, Pearson correlation coefficient and regression analysis were used to compare quantitative variables.

\section{Results}

The results showed that 86 of participants $(58.1 \%)$ were female and 62 (41.9\%) were male; 109 (73.6\%) had age $60-75$ years and the rest aged $>75$ years; $102(68.9 \%)$ were married and the rest were divorced or widowed; 114 (77\%) were unemployed; 123 (83.1\%) were living in rural areas; $120(81 \%)$ are illiterate and $5(3.3 \%)$ had high school diplomas or higher degree; 77 patients $(52 \%)$ had no depression, 57 (38.5\%) moderate depression and $14(9.5 \%)$ had severe depression. In overall, $48 \%$ had depressive symptoms. In terms of ADL, $97.3 \%$ were independent and in terms of IADL, only $58.8 \%$ were independent.

Spearman correlation test was used to investigate the relationship between depression variable and instrumental/noninstrumental daily living activities. According to the results, there was a negative and significant relationship between them, meaning that depression decreases with increasing instrumental and non- instrumental daily living activities (Table 1). In order to determine the predictors of depression, a linear regression model was used which included depression as a response variable while instrumental/non-instrumental daily activities, gender, marital status, place of residence, age, and education were considered predictor variables. The value of $\mathrm{R}^{2}$ value in this model was 0.221 , indicating that $22.1 \%$ of the scatter observed in this model is explained by these seven variables. The variables of instrumental/noninstrumental daily activities, gender and marital status were significant $(\mathrm{P}<0.05)$, but the variables of education, place of residence and age were not significant $(\mathrm{P}>0.05)$.

Depression $=3.317+A D L-0.12+I A D L+-0.43$ gender + -0.355 marital status

\section{Conclusion}

Depression was inversely related to instrumental/non-instrumental daily living activities in the elderly. Hence, it can be said that the ability of the elderly to perform daily living activities (with and without instruments) is a good predictor of depression in them. By carefully examining the health of the elderly and their ability by health care teams and their family members, it is possible to prevent the development of their inability in perform daily living activities, as well as their depression.

Table 1. Correlation of depression with instrumental/non-instrumental daily activities

\begin{tabular}{llll}
\hline & Variable & ADL & IADL \\
\hline \multirow{2}{*}{ Depression } & Pearson correlation $(r)$ & -0.193 & -0.304 \\
& Sig. & 0.019 & $>0.001$ \\
\hline
\end{tabular}




\section{Ethical Considerations}

Compliance with ethical guidelines

All ethical principles are considered in this article. The participants were informed about the purpose of the research and its implementation stages. They were also assured about the confidentiality of their information and were free to leave the study whenever they wished, and if desired, the research results would be available to them.

\section{Funding}

This study was extracted from a research proposal and received financial support from the Deputy for ResearchAnd Technology of North Khorasan University of Medical Science.

\section{Authors' contributions}

Conceptualization: Marzieh Mohamadzadeh, Vahid Rashedi; Methodology: Vahidreza Borhaninejad, Mitra Hashemi; Investigation: Mitra Hashemi, Marzieh Mohamadzadeh; Writing original draft: Marzieh Mohamadzadeh, Vahid Rashedi; Review \& editing: All author.

\section{Conflicts of interest}

The authors declared no conflict of interest. 


\title{
بررسى ارتباط بين فعاليتهاى روزانه زندگى و افسردگى در سالمندان شهرستان مانه و سملقان
}

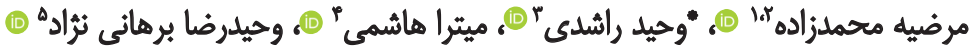 \\ ا. مركز بهداشت مانه و سملقان، دانشعاه علوم يزشكى خراسان شمالى، بجئورد، ايران.

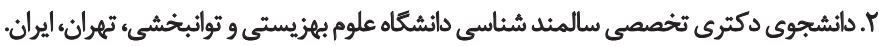

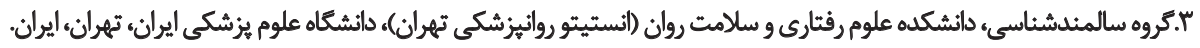

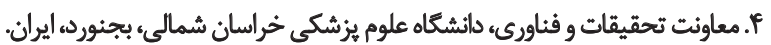

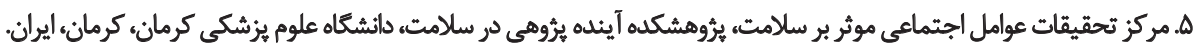

\begin{abstract}
حكند

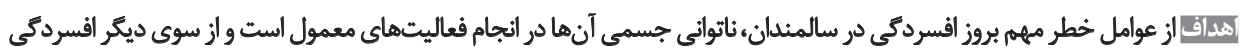

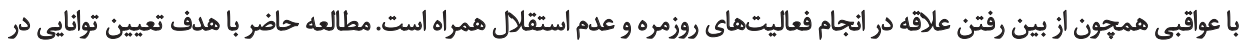

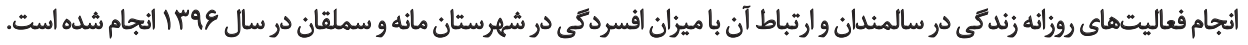

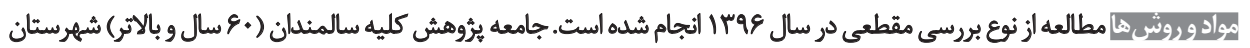

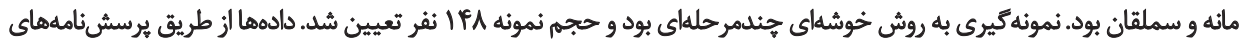

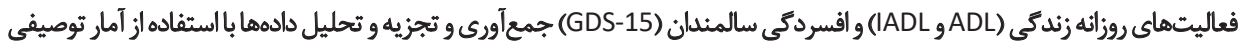

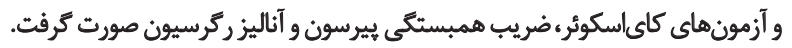

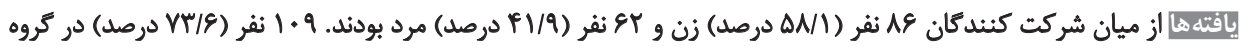

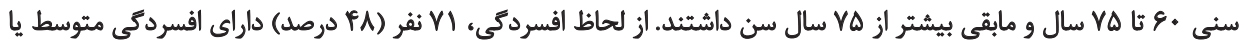

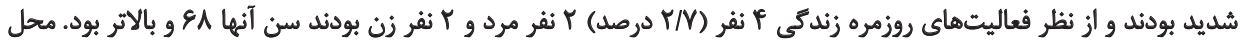

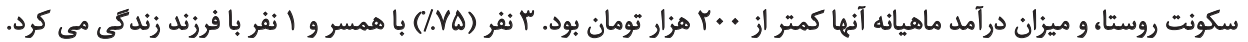

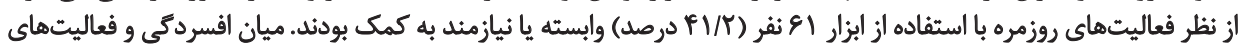

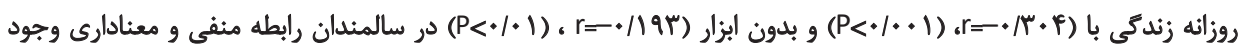

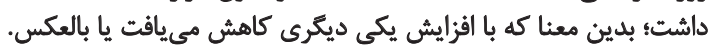

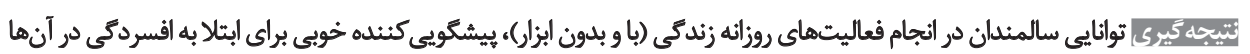

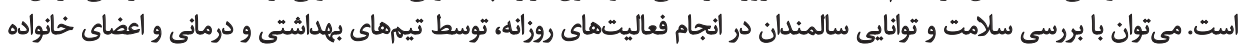

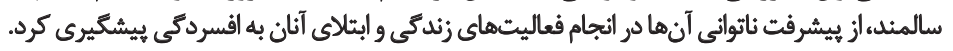

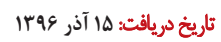

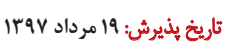

جمعيت بود،لذا ايران در زمره كشورهاى در حال سالمئد شدن

قرار كرفته است [ب].

dates

با سالمند شدن ملتها، مشكلات سالمندان به طور روزافزون إنهان

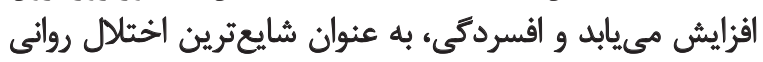

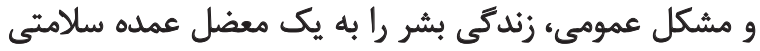

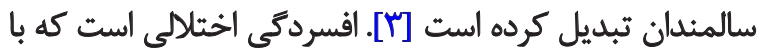

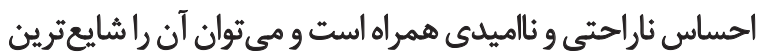

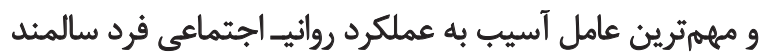

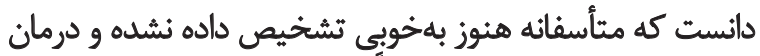

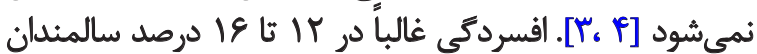

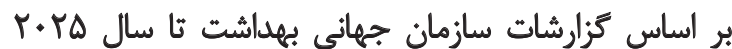

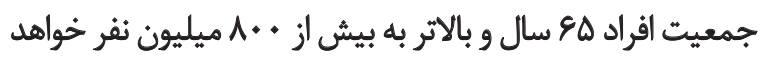

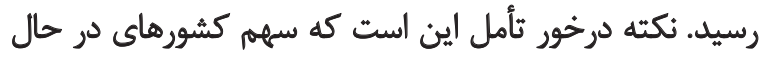

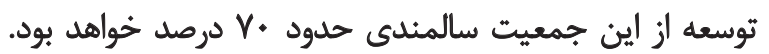

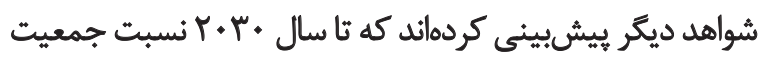

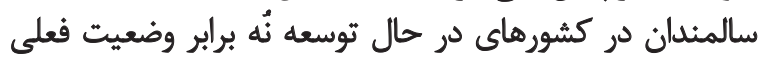

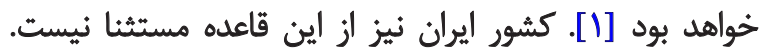

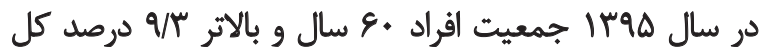

\section{$-$}

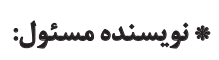

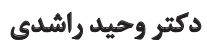

نشائى: تهران، دانشكاه علوم يزشكى ايران، دانشكده علوم رفتارى و سلامت روان (انستيتو روانيزشكى تهران)، كروه سالمثلشثاسى.

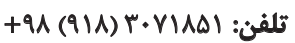
vahidrashedi@yahoo.com; rashedi.v@iums.ac.ir بست الكترونيكي 


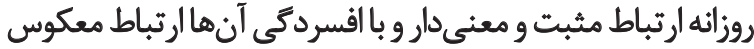

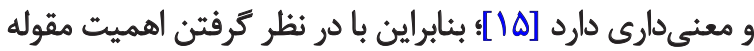

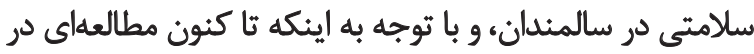

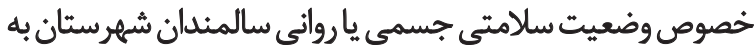

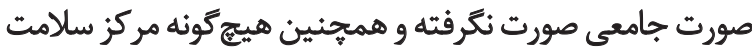

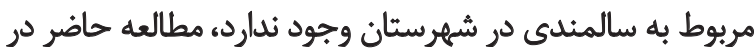

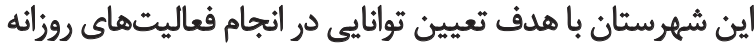

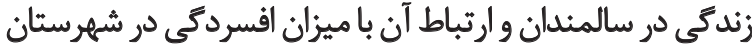
مانه و سملقان در سال عوبـا انجام شده است.

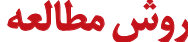

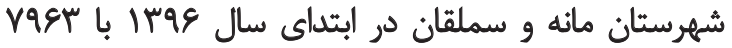

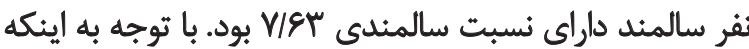

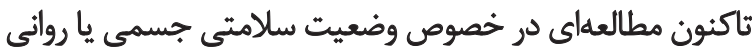

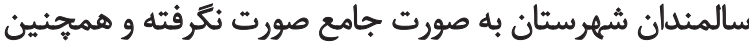

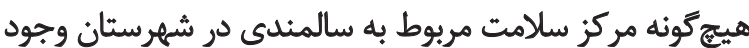

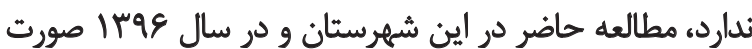

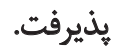

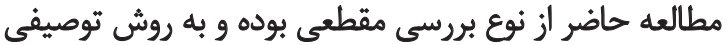

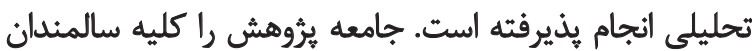
(افراد • ع سال و بالاتر) شهرستان مانه و سملقان تشكيل مي دادئد.

روش نمونه كيرى بدين صورت بود كه با توجه به وجود با مركز

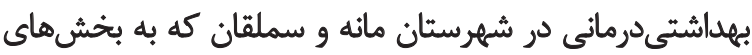

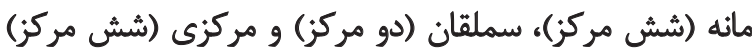

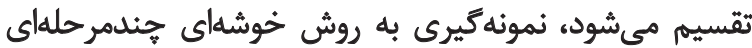

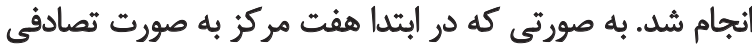

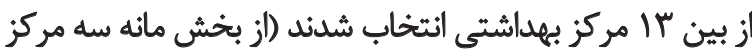

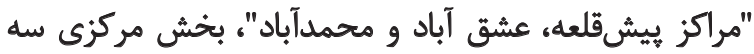

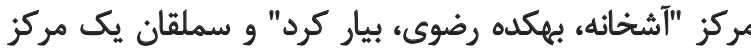

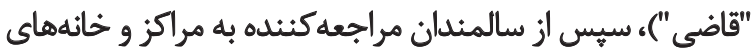

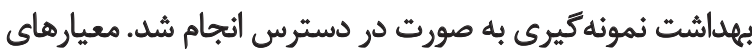

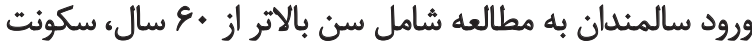

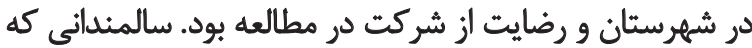

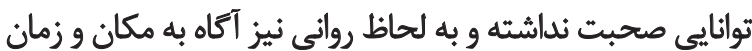
نبودنده از مطالعه خارج شدند.

به دليل بعسوادى يا سطح سواد بايين سالمندان منطقه

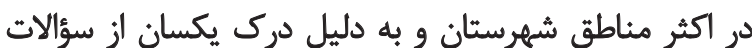

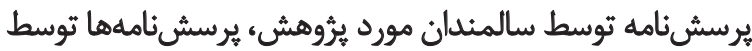

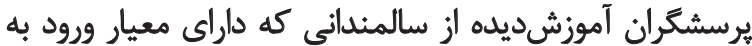
مطالعه بودند تكميل شدر.

جهت برآورد حجم نمونه، به اين دليل كه ثاكنون مطالعهاى

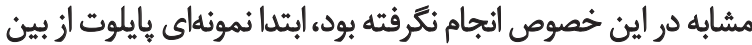

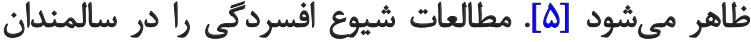

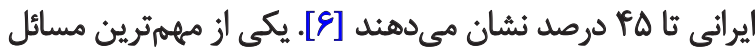

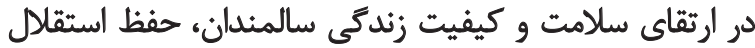

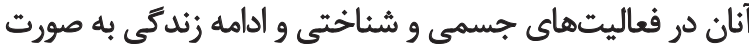

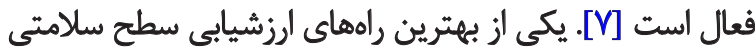

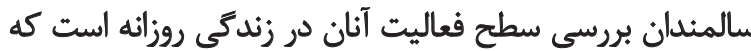

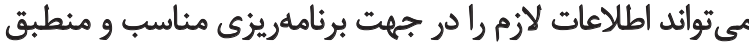

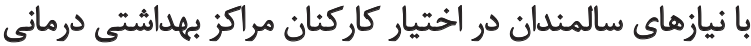

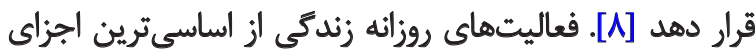

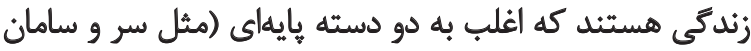

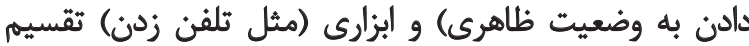

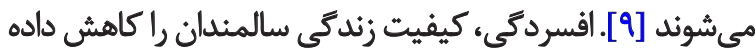

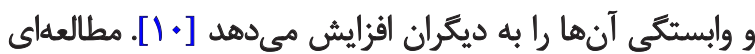

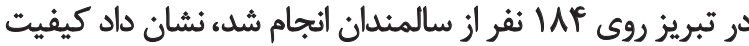

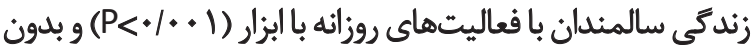

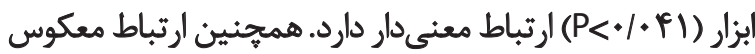

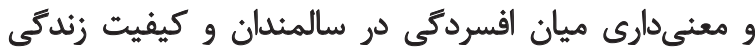

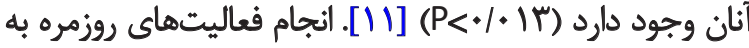

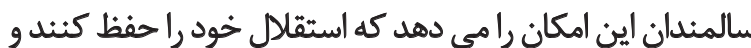

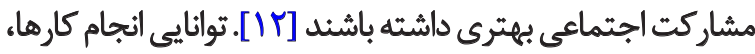

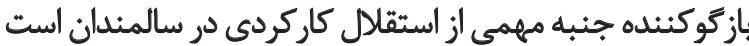

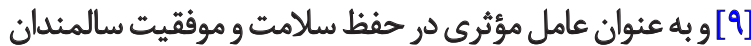

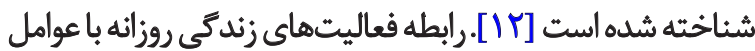

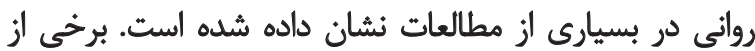

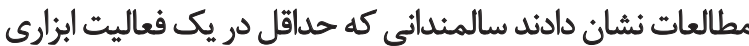

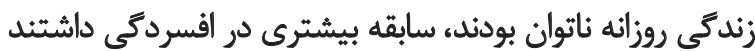

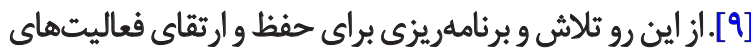

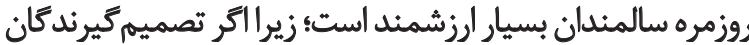

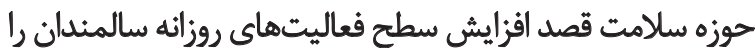

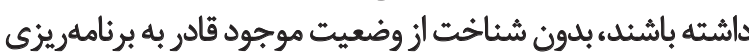

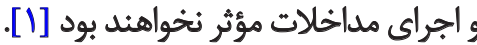

با توجه به اهداف كلى يزشكى در بيشكيرى و ارتقاى سلامت دامت

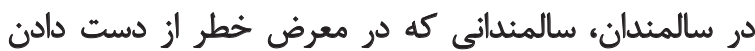

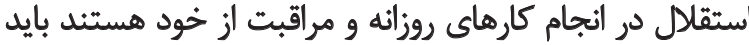

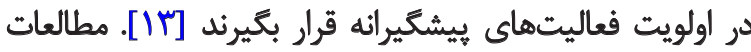

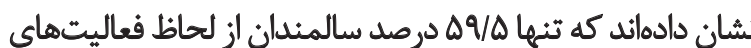

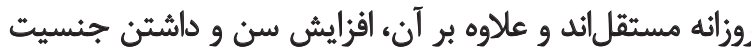

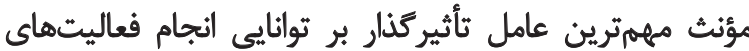

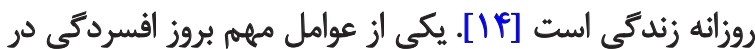

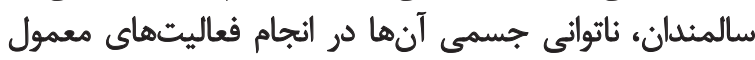

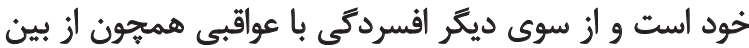

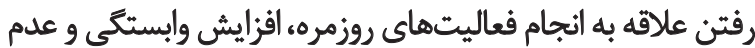

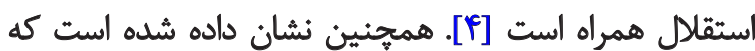

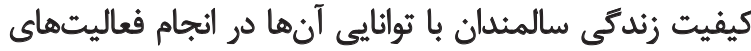


و خير است و دستهبندى افراد بدين صورت است كه اكر فردى إمي

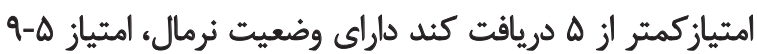

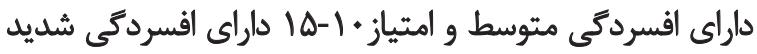

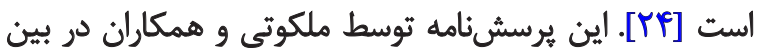

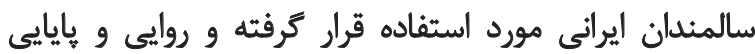

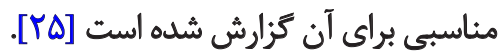

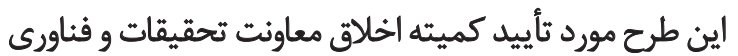

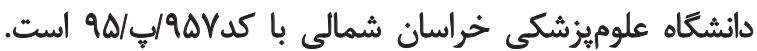

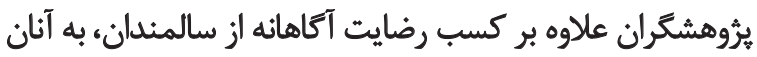

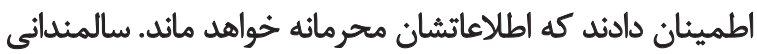

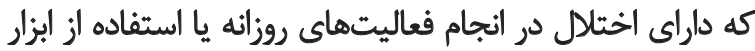

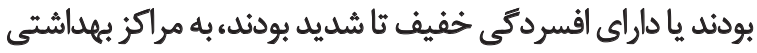

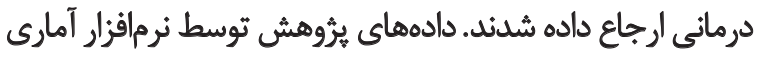

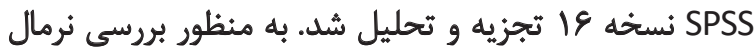

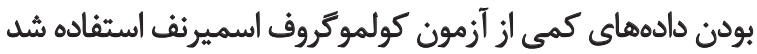

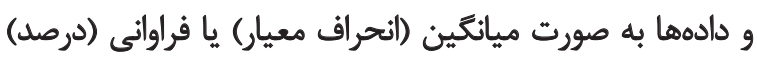

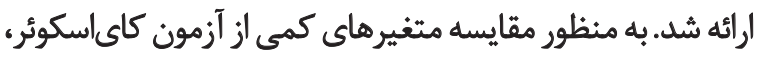

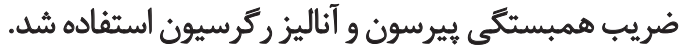

يافتهها

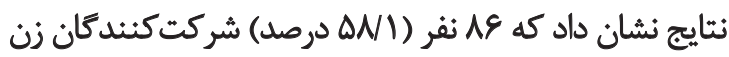

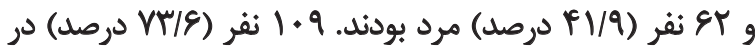

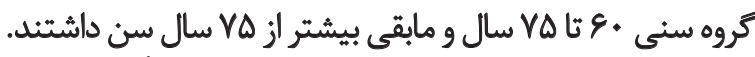

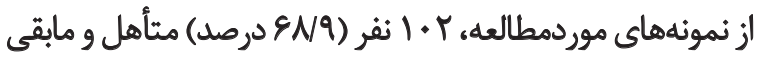

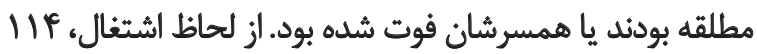

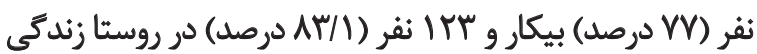

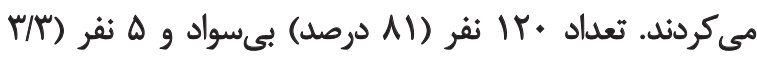

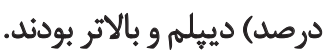

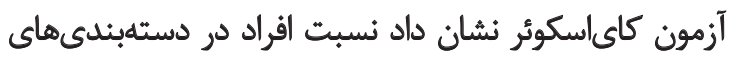

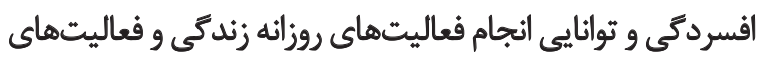

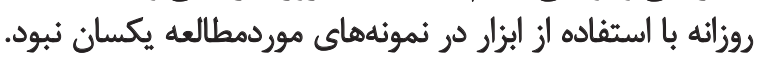

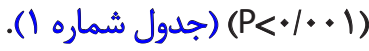

به منظور بررسى ارتباط بين متغير افسردگى با فعاليتهاى

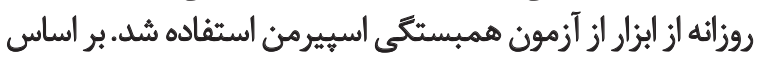

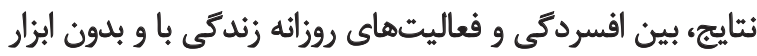

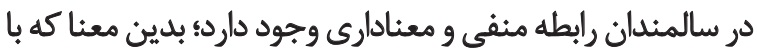

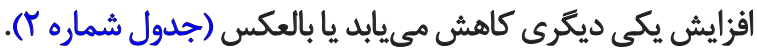

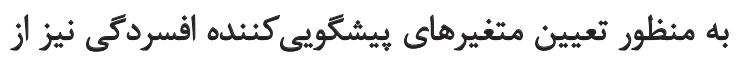

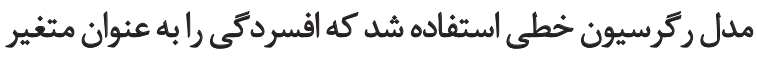

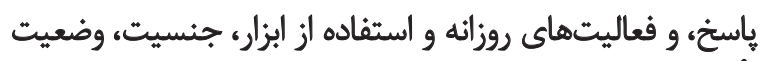

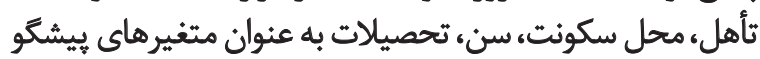

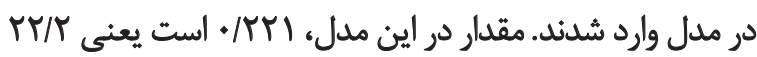

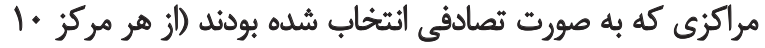

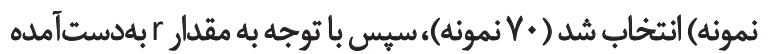

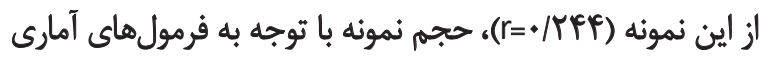

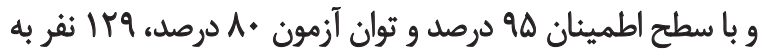

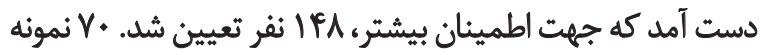
وايلوت جزو أ ا نفر نمونه لازم در نظر كرفته شدند.

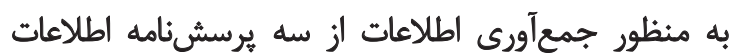

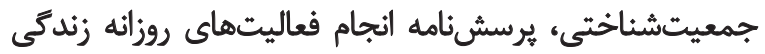

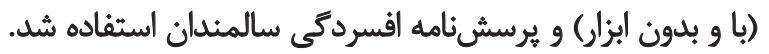

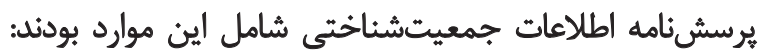
سن، جنس، تأهل، سطح تحصيلات، درآهد.

به منظور سنجش توانايي در انجام فعاليتهاى روزمره

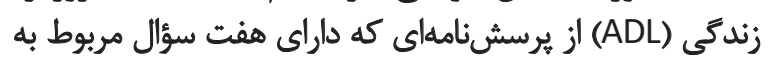

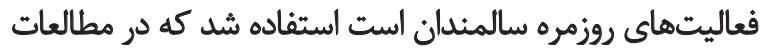

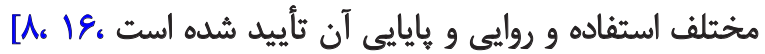

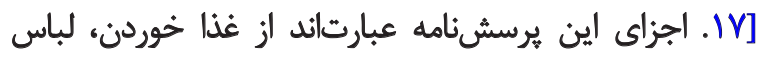

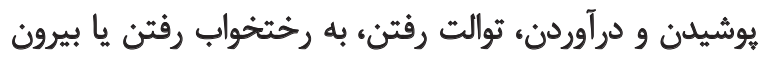

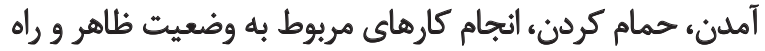

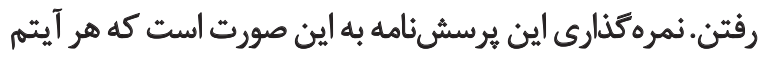

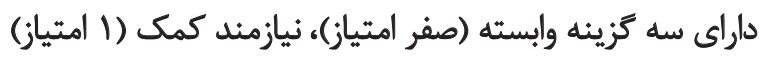

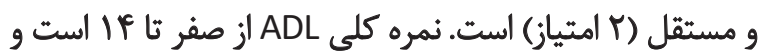

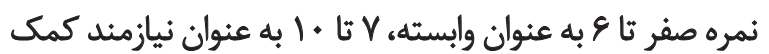

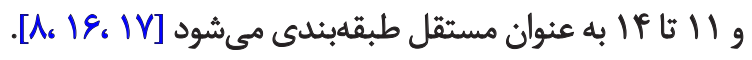
به منظور سنجش توانايى فعاليتهاى روزانه با استفاده از ابزار

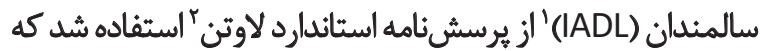

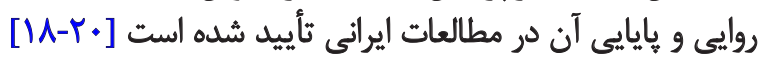

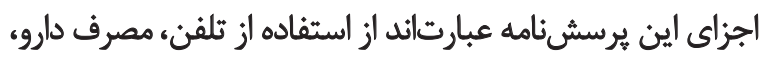

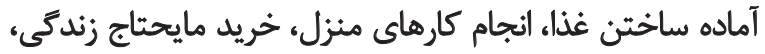

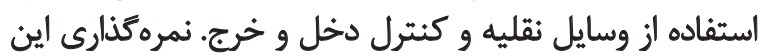

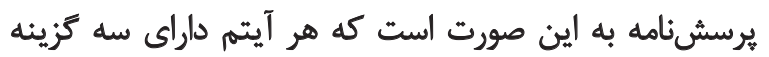

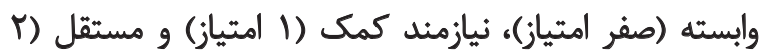

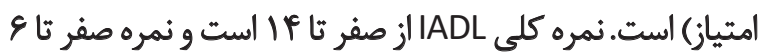

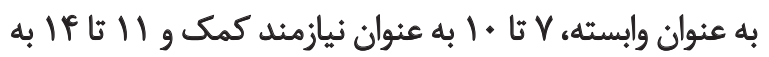
عنوان مستقل طبقهبندى مىشود [19].

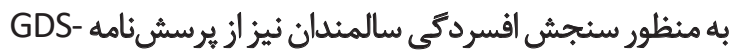

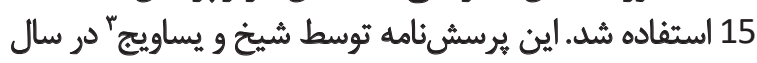

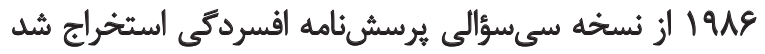

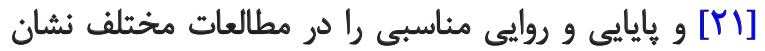

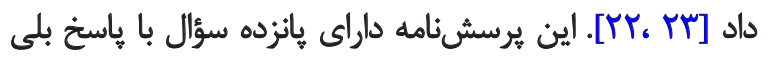

1. Instrumental activities of daily living

2. Lawton

3. Sheikh \& Yesavage 
جدول ا. وضعيت افسردكى، فعاليتهاي روزمره زندكى و فعاليتهاي روزائه زندكى با استفاده از ابزار در سالمندان

\begin{tabular}{|c|c|c|c|}
\hline سطع معنى دارى أزمون كاىاسكوئر & فراوانى (درصد) & طبقدبثدى & نام متغير \\
\hline \multirow{3}{*}{$<+1+\infty$} & $W(\Delta T)$ & بلدون افسردكى (t-fis) & \multirow{3}{*}{ وضعيت افسردكى } \\
\hline & $\Delta V(T N \Delta)$ & افسردگى متوسط (9-a) & \\
\hline & If $(9 / \Delta)$ & افسردمى شديد (10-1.10) & \\
\hline \multirow{3}{*}{$<\cdot 1 \cdot .1$} & Iffe $\left(9 V / T^{2}\right)$ & هسثقل (1)-11) & \multirow{3}{*}{ فعاليتهاي روزمره زندكى } \\
\hline & $r(T)$ & نيازمند كمى (·-1) & \\
\hline & $1(\cdot M)$ & وابسته (ع-•) & \\
\hline \multirow{3}{*}{$<+1 *+1$} & $\operatorname{Ar}(\Delta N A)$ & مسثقل (1)-11) & \multirow{3}{*}{ فعاليتهاى روزمره زندكى با اسثفاده از ابئزر } \\
\hline & $P(r q / V)$ & نيازمند كمى (•l- (V- & \\
\hline & $\operatorname{IV}(11 / \Delta)$ & وابسته (8-•) & \\
\hline
\end{tabular}

جدول Y. همبستكى افسردّى، فعاليتهاى روزمره زندكى و فعاليتهاى روزانه زندكى با استفاده از ابزار در سالمثدان

\begin{tabular}{|c|c|c|c|}
\hline فعاليتهاى روزمره زندكى با استفاده از ابزار & فعاليتهاي روزمره زندكى & \multicolumn{2}{|c|}{ 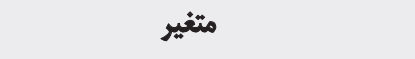 } \\
\hline$-* / r \cdot 4$ & $-+/ 19 \%$ & ضريب همبستكى & اففس رك \\
\hline$<+1 .+1$ & $.1+19$ & سطح معنادارى & s. \\
\hline
\end{tabular}

il

جدول ஈ. ركرسيون خطى تعيين ثأثير فعاليتهاى روزائه و استفاده از ابزار بر افسردكى

\begin{tabular}{|c|c|c|c|c|c|}
\hline سطح معنى دارى & أماره آزمون & ضريب ركرسيون استانداردشده & خطاى معيار & ضريبرّكرسيون & مثغيرها \\
\hline$<\cdot / *+r$ & $m \cdot M$ & & $1 / .99$ & rir & عرض از مبلا - (y-intercept) \\
\hline.$+P$ & $-r / \bullet \cdot V$ & $-\cdot / 1 r^{n}$ & +1.8 & $-+/ 1 r$ & فعاليتهاى روزمره زندكى \\
\hline .1 .1 & $-r / 818$ &.$- / M \Delta \Delta$ & $.1 \cdot 18$ & $-*+\pi r$ & فعاليتهاى روزمره زئدكى با استفاده \\
\hline .1 .01 & r/mPa &.$/ T r T$ &.$/ 1 \mathrm{re}$ &.$/ \pi$ & جنسيت \\
\hline .1 .18 & - & $-* / r \cdot 9$ &.$/ 148$ &.$- / T \Delta \Delta$ & وضعيت تأهل \\
\hline.$/ \Delta \Delta \Delta$ &.$- / 1094$ &.$- / .48$ & .1189 & $-\bullet / 1$ & وضعيث سواد \\
\hline.$/ 109$ & $-1 / 410$ & $-* / 1 \cdot 9$ &.$/ T \Delta A$ &.$- /$ reg & محل سكونت \\
\hline $.1 . e r$ & I/Av9 &.$/ 101$ & $\cdot 1 \cdots 1$ & $.1+10$ & سن سن \\
\hline
\end{tabular}

درصد از يراكندكى مشاهدشده در اين مدل توسط اين هفت

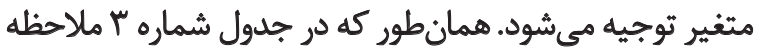

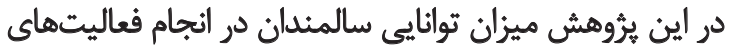

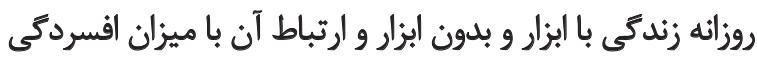

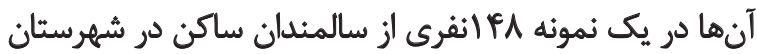

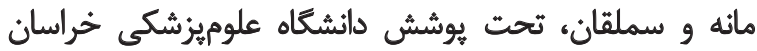

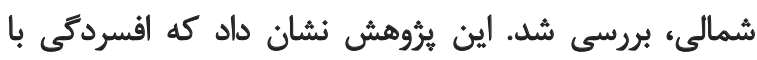

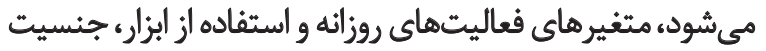

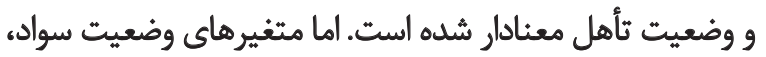

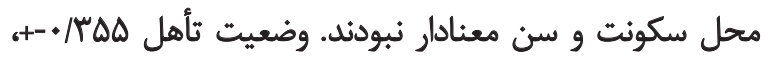

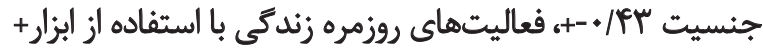

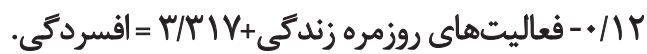

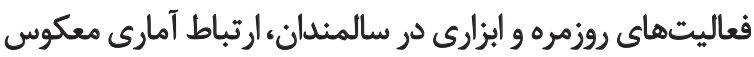

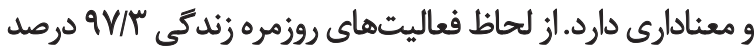


اندازمكيرى باشد. نتايج مطالعات مشابه در كشور نشان مى دهد

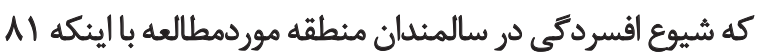

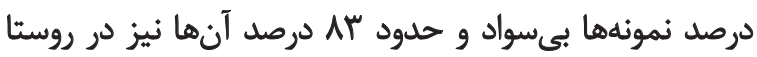
زندكى مي كردند بيشتر از ساير مناطق كشور نيست نيست نتايج نشان

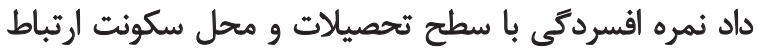

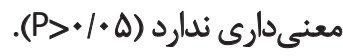

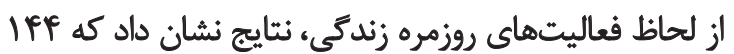

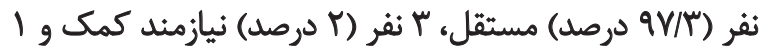

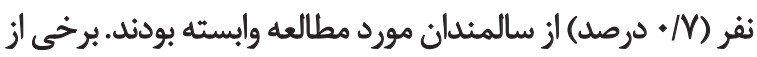

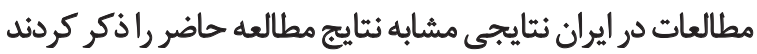

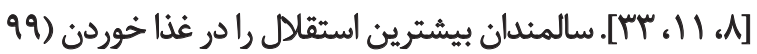

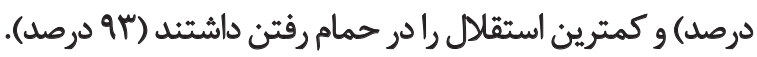

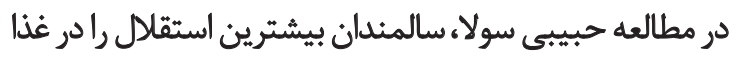

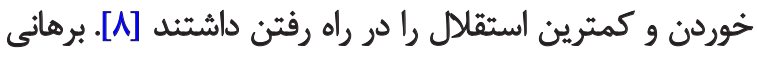

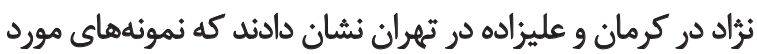

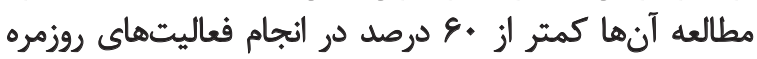

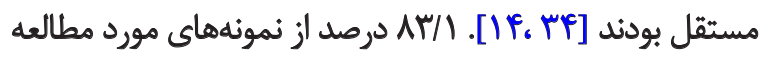

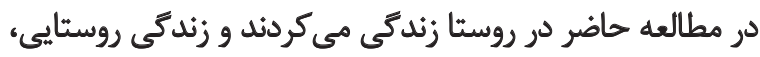

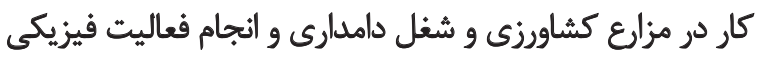

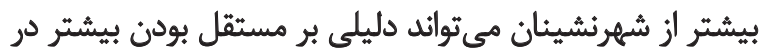

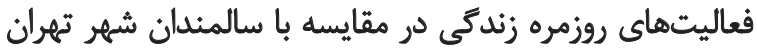

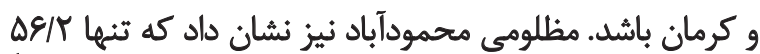

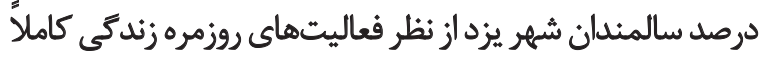

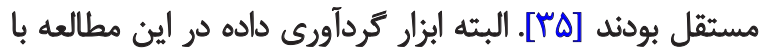

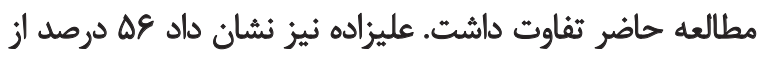

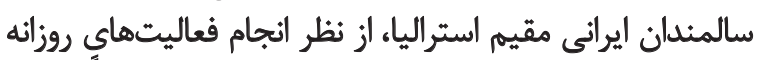

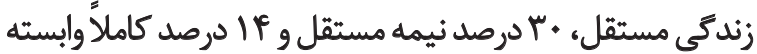

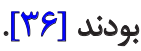

فعاليتهاى ابزارى زندكى در سالمندان مستلزم داشتن

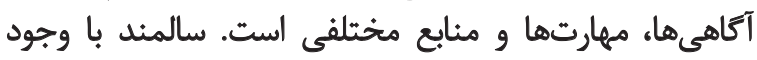

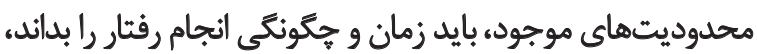

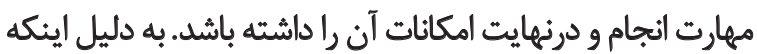

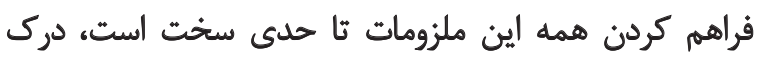

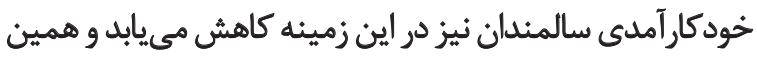

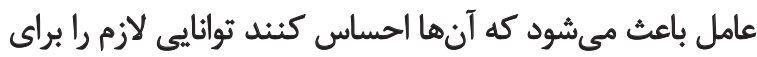

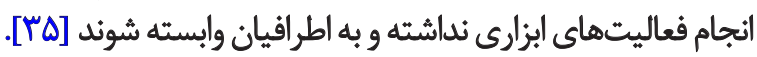

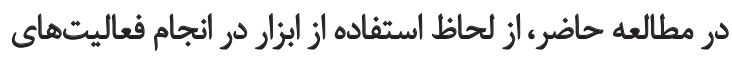

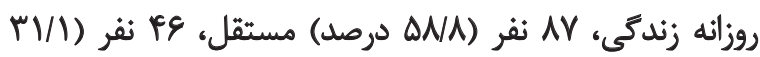

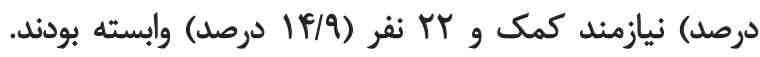

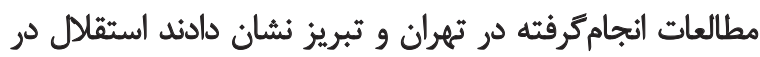

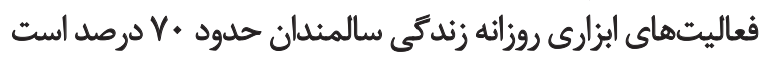

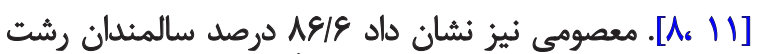

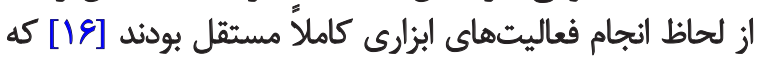

مستقل و از لحاظ استفاده از ابزار در انجام فعاليتهاى روزانه

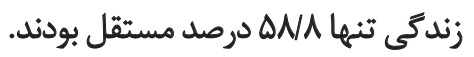

افسردگى با فعاليتهاى روزمره و ابزارى در سالمندان، ارتباط

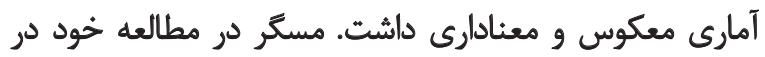

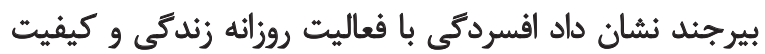

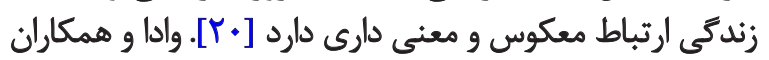

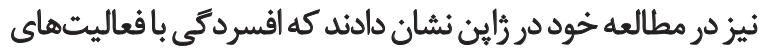

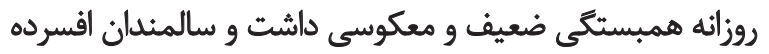

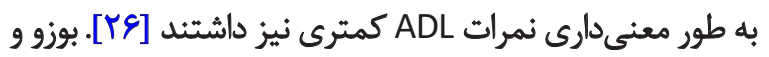

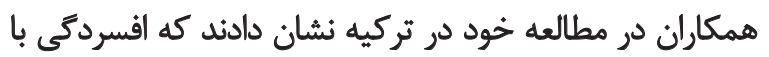

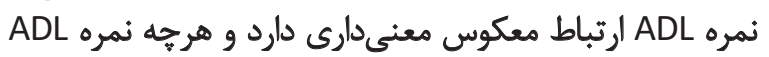

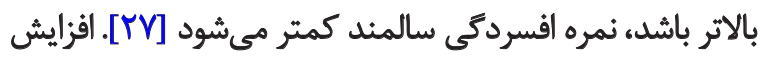

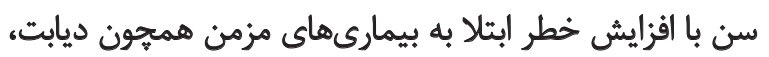

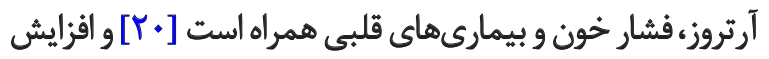

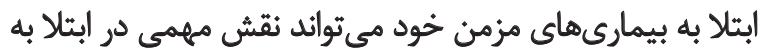

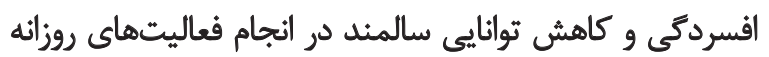

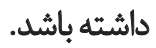

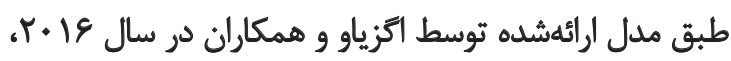

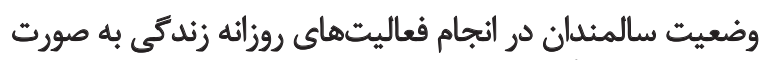

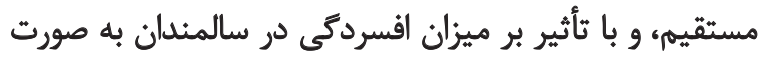

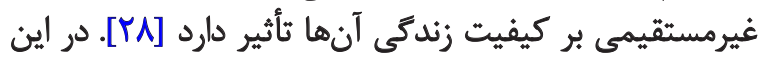

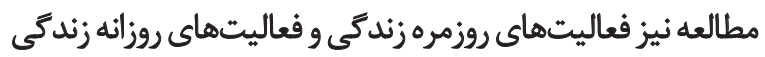

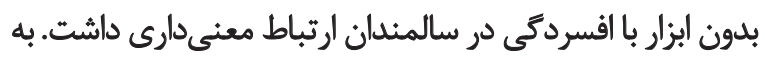

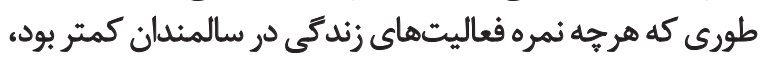

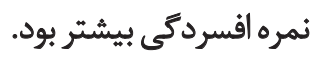

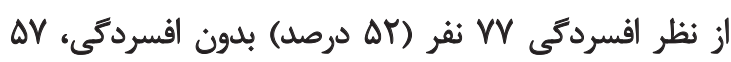

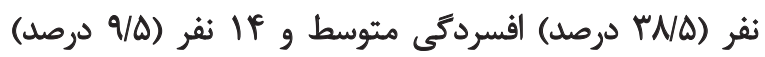

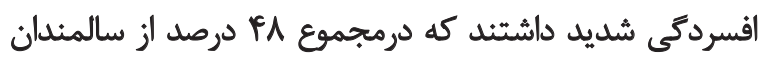

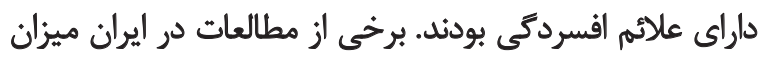

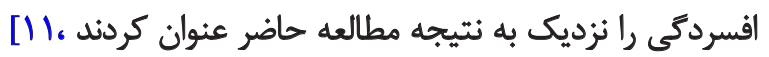

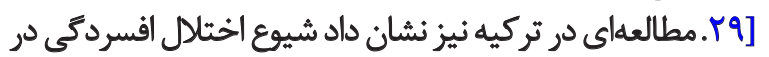

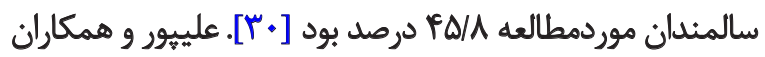

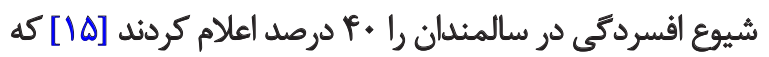

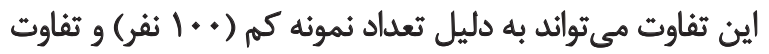

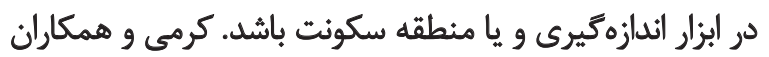

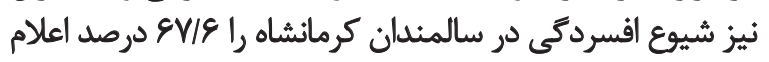

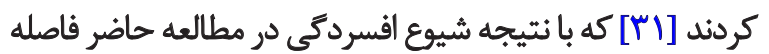

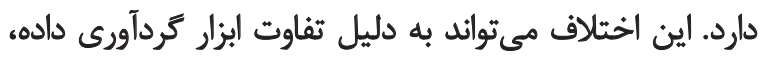
تفاوتهاى قومى و منطقهاي باشد.

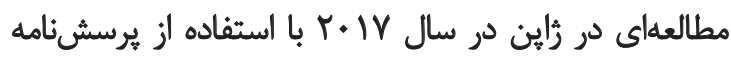

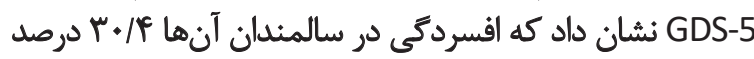

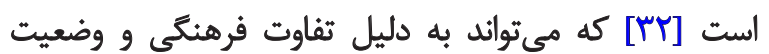

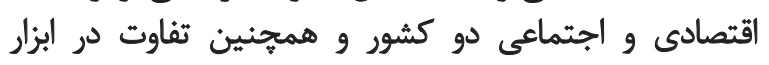


مشاركت نويسندكًان

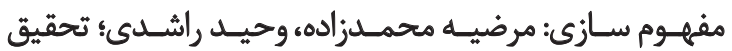

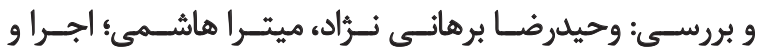

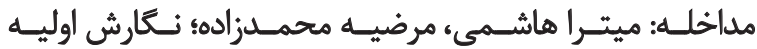

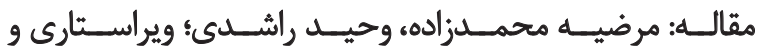

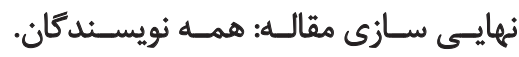

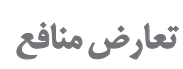

نويسندكان مقاله هيج كُونه تضاد منافعى ندارند

$$
\text { تشكر وقدرداني }
$$

محققين بر خود لازم مي دانثد از معاونت محترم تحقيقات و روائ

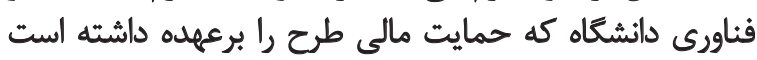

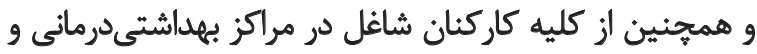

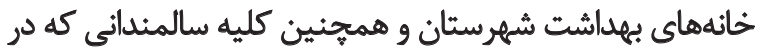
انجام اين بثروهش همكارى كردند، تقدير و تشكر كنئد.
با نتايج مطالعه حاضر تفاوت دارد. در مطالعه حاضر فعاليتهاى

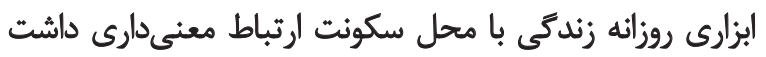

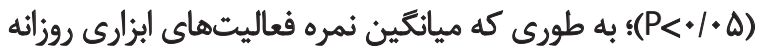

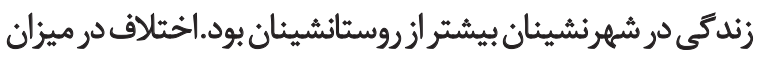

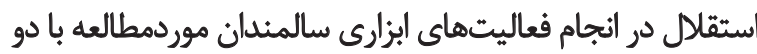

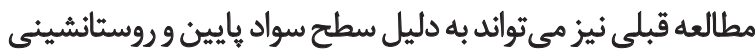

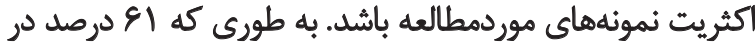

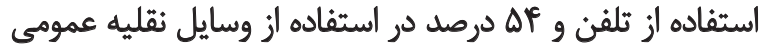

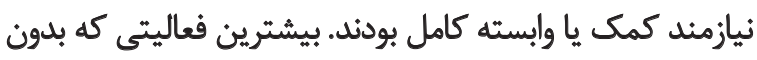

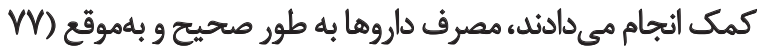

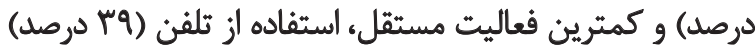

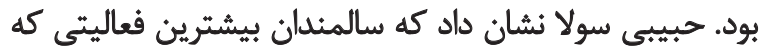

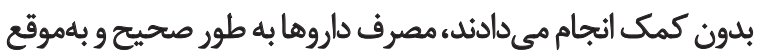
و كمترين مورد انجام تعميرات جزئي منزل بود [A]].

\section{نتيجلكيرى نهمايي}

توانايى سالمندان در انجام فعاليتهاى روزانه زندكى (باو بدون

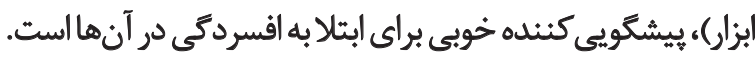

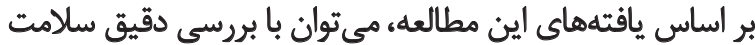

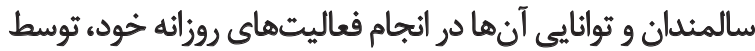

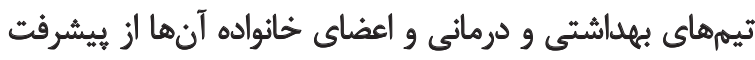

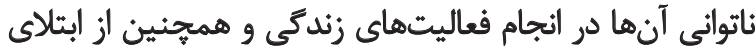

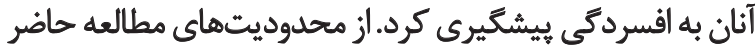

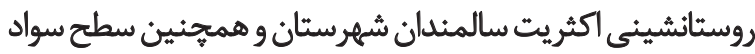

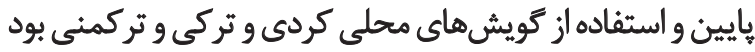

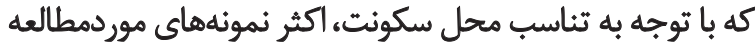

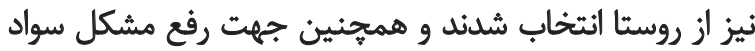

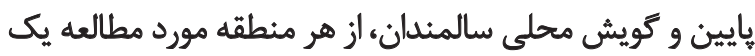

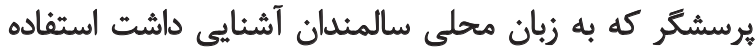

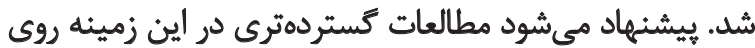

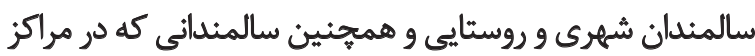

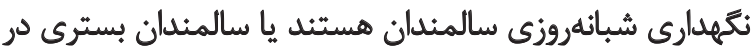
بيمارستان در ساير نقاط كشور انجام شود.

\section{مالاحظات اخلاقي}

\section{ييروى از اصول اخلاق ثئوهش}

اين مقاله حاصل طرح تحقيقاتيى مصوب معاونت تحقيقات و

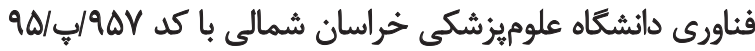
است.

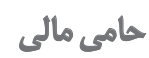

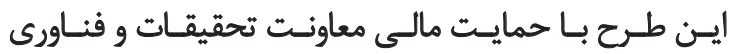

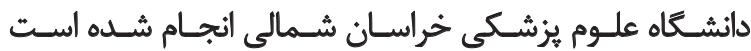




\section{References}

[1] Taheritanjani P, Azadbakht M. [Psychometric properties of the Persian version of the activities of daily living scale and instrumental activities of daily living scale in elderly (Persian)]. Journal of Mazandaran University of Medical Sciences. 2014; 132(25):103-13. http://jmums.mazums.ac.ir/article1-6766-en.html

[2] Allahyari E, Keramati M, Kamali M. [Predicting elderly depression prevalence in different Iranian ethnicities and associated factors (Persian)]. Salmand: Iranian Journal of Ageing. 2020; 15 (1):129-18. http://salmandj.uswr.ac.ir/artii cle-1-1915-fa.html

[3] Hekmatipoor N, Hojati H, Sharifnia H, Akhondzadeh G, Nikjoo A, Mirabilhasani M. [Effect of exercise on depression in elderly (Persian)]. Journal of Health Building Community Health Education. 2012; 1(3):23-32. http://journal.ihepsa.ir/ article-1-66-en.html

[4] Ghaderi S, Sahaf R, Mohammadi Shahbalaghi F, Ansari G, Gharanjic A, Ashrafi K, et al. Prevalence of depression in elderly Kurdish community residing in Boukan, Iran. Iranian Journal of Ageing. 2012; 7(1):57-66. https://www.sid.ir/en/ journal/ViewPaper.aspx?ID=562617

[5] Asghari N, Aliakbari M, Dadkhah A. [The effects of group Logotherapy on decreasing the degree of depression in female olds (Persian)]. Middle Eastern Journal of Disability Studies. 2012; 11(2):31-8. http://jdisabilstud.org/article-1-311-en.html

[6] Rashedi V, Rezaei M, Foroughan M, Delbari A. Validity and reliability of the Depression In Old Age Scale (DIA-S) in Iranian older adults. Archives of Gerontology and Geriatrics. 2016; 66:193-7. [DOI:10.1016/j.archger.2016.06.009] [PMID]

[7] Rashedi V, Asadi-lari M, Foroughan M, Delbari A, Fadayeva$\tan$ R. Prevalence of disability in Iranian older adults in Tehran, Iran: A population-based study. Journal of Health and Social Sciences. 2016; 1(3):251-62. https://journalhss.com/wp-content/uploads/JHHS13_251-262.pdf

[8] Habibisola A, Nikpour S, Seiedshohada M, Haghani H. [Quality of life and statuse of physical functioning among elderly people in west region of Tehran: A cross-sectional survey (Persian)]. Iranian Journal of Nursing. 2006; 21(53):29-39. http:/ /ijn. iums.ac.ir/article-1-406-en.html

[9] Khajavi D, Parsa Z. The Development of Psychometric Properties of "the elderly's activities of daily life scale". Journal of Development and Motor Learning. 2014; 6(1):91-108. [DOI:10.22059/JMLM.2014.50192]

[10] Sherina M, Rampel L, Mostaqim A. The prevalence of depression among the elderly in Sepang, Selangor. Medical Journal of Malaysia. 2004; 59(1):45-9. [PMID]

[11] Payahoo L, Khaje-bishak Y, Poorghasem B, Asghari Jafarabad M, Kabir-alavi M. [The survey of the relationship between quality of life of elderly with depression and physical activity in Tabriz, Iran (Persian)]. Scientific Journal of Rehabilitation Medicine. 2011; 2(2):39-46. [DOI:10.22037/JRM.2013.1100142]

[12] Rashedi V, Gharib M, Yazdani AA. Social participation and mental health among older adults in Iran. Iranian Rehabilitation Journal. 2014; 12(1):9-13. https://applications.emro.who. int/imemrf/Iran_Rehabil_J/Iran_Rehabil_J_2014_12_19_9_13. pdf
[13] Nazari H, Rashedi V, Mohammadi H, Yousefi M. [Relationship between cognitive status and activities of daily living among the elderly of nursing homes (Persian)]. Journal of Kermanshah University of Medical Sciences. 2015; 18(12):744-6. [DOI:10.22110/jkums.v18i12.2390]

[14] Alizadeh M, Rahimi A, Arshinchi M, Sharifi F, Arzaghi Seyed M, Fakhrzadeh H. [Physical health status and socio-economic outcomes on elderly in Tehran metropolitan area (Persian)]. Journal of Diabetes and Metabolism of Iran. 2013; 13(1):29-38. http:/ / ijdld.tums.ac.ir/article-1-5089-en.html

[15] Alipour F, Sajadi H, Forouzan A, Nabavi H, Khedmati E. [The Role of Social Support in Reducing Anxiety and Depression in the Elderly (Persian)]. Iranian Journanl of Ageing. 2009; 4(1):53-61. http://salmandj.uswr.ac.ir/article-1-333-en.html

[16] Masoumi N, Jafroudi S, Ghanbari Khanghah A, Kazemnejad Leili E. [Assessment of retired elderly's people autonomy and its affecting factors in Rasht. Journal of Holistic Nursing And Midwifery (Persian)]. 2011; 21(1):46-51. http://hnmj.gums.ac.ir/ article-1-193-en.html

[17] Habibi A, Savadpoor M, molaei B, SHamshiri M, Ghorbani M. Survey of physical functioning and prevalence of chronic illnesses among the elderly people. Iranian Journal of Ageing. 2007; 4(3):67-78. http://salmandj.uswr.ac.ir/article-1-314-en.html

[18] Hassani Mehraban A, Soltanmohamadi Y, Akbarfahimi M, Taghizadeh G. Validity and reliability of the persian version of lawton instrumental activities of daily living scale in patients with dementia. Medical Journal of the Islamic Republic of Iran. 2014; 28:25. [PMID] [PMCID]

[19] Taheri Tanjani P, Azadbakht M. [Psychometric properties of the Persian version of the activities of daily living scale and instrumental activities of daily living scale in elderly (Persian)]. Journal of Mazandaran University of Medical Sciences. 2016; 25(132):103-12. http://jmums.mazums.ac.ir/article-1-6766-en. html

[20] Mesgar S, Amini Nasab Z, Nakhaei MH, Sharifzadeh G, Javadinia SA. [Study of quality of life, depression, and daily routines in rural elders in Birjand city, Iran, in 2013 (Persian)]. Iranian Journal of Ageing. 2015; 10(3):142-7. http:/ / salmandj.uswr.ac.ir/ article-1-753-fa.html

[21] Shiekh J. Geriatric Depression Scale (GDS): Recent evidence and development of a shorter version. Journal Clinical Gerontologist. 1986; 5(1-2):165-73. [DOI:10.1300/J018v05n01_09]

[22] Boey KW. The use of GDS-15 among the older adults in Beijing. Journal Clinical Gerontologist. 2000; 21(2):49-60. [DOI:10.1300/ J018v21n02_05]

[23] Teh EE, Hasanah CI. Validation of Malay version of Geriatric Depression Scale among elderly inpatients. Age. 2004; 17:65.4. http:// eprints.usm.my/10198/1/Validation_of_Malay_Version_of_Geriatric_Depression_Scale_Among_Elderly_Inpatients_(PPSPerubatan).pdf

[24] Mirzaei M, Sahaf R, Mirzaei S, Sepahvand E, Pakdel A, Shemshadi $\mathrm{H}$. [Depression and its associated factors in elderly nursing home residents: A screening study in Khorramabad (Persian)]. Iranian Journal of Ageing. 2015; 10(1)54-61. http://salmandj. uswr.ac.ir/article-1-761-en.html

[25] Malakouti SK, Fatollahi P, Mirabzadeh A, Salavati M, Zandi T. Reliability, validity and factor structure of the GDS_15 in Iranian elderly. International Journal of Geriatric Psychiatry. 2006; 21(6):588-93. [DOI:10.1002/gps.1533] [PMID] 
[26] Wada T, Ishine M, Sakagami T, Okumiya K, Fujisawa M, Murakami $\mathrm{S}$, et al. Depression in Japanese community-dwelling elderly-prevalence and association with ADL and QOL. Archives of Gerontology and Geriatrics. 2004; 39(1):15-23. [DOI:10.1016/j. archger.2003.12.003] [PMID]

[27] Bozo Ö, Toksabay NE, Kürüm O. Activities of daily living, depression, and social support among elderly Turkish people. The Journal of Psychology. 2009; 143(2):193-206. [DOI:10.3200/ JRLP.143.2.193-206] [PMID]

[28] Xiao H, Yoon JY, Bowers B. Quality of life of nursing home residents in China: A mediation analysis. Nursing \& Health Sciences. 2017; 19(2):149-56. [DOI:10.1111/nhs.12288] [PMID]

[29] Bakhtiari M, Emaminaeini M, Hemati H, Khodakarami S, Sahaf R. Depression and perceived social support in the Tehran. Iranian Journanl of Ageing. 2017; 12(2):192-207. [DOI:10.21859/ sija-1202192]

[30] Arslantas D, Ünsal A, Ozbabalık D. Prevalence of depression and associated risk factors among the elderly in Middle Anatolia, Turkey. Geriatrics \& Gerontology International. 2014; 14(1):100-8 [DOI:10.1111/ggi.12065] [PMID]

[31] Karami N, Rezaei J, Jozanifar Y, Abdi M, Aghaei A, Astanegi S, et al. [A survey of the depression rate among the elderly in Kermanshah, 2012 (Persian)]. Journal of Clinical Research in Paramedical Sciences. 2016; 5(1):23-30. https:// sites.kowsarpub.com/ jcrps/articles/81443.html

[32] Nakamura T, Michikawa T, Imamura H, Takebayashi T, Nishiwaki Y. Relationship between depressive symptoms and activity of daily living dependence in older Japanese: The Kurabuchi study. Journal of the American Geriatrics Society. 2017; 65(12):2639-45. [DOI:10.1111/jgs.15107] [PMID]

[33] Joghataei M, Nejati V. [Assessment of health status of elderly people in the city of Kashan (Persian)]. Iranian Journal of Ageing. 2006; 1(1):3-10. http://salmandj.uswr.ac.ir/article-1-26-fa.html

[34] Borhaninejad V, Momenabadi V, Hosseini S, Mansori T, Sadeghi A, Toroski M. Health physical and mental status in the elderly of Kerman. Journal of North Khorasan University. 2015; 6(4):715-23. [DOI:10.29252/jnkums.6.4.715]

[35] Mazloomymahmmodabad S, Soltani T, Morowatisharifabad M, Fallahzadeh H. [Activities of daily living and prevalence of chronic disease among elderly people in Yazd (Persian)]. Toloo-EBehdasht . 2014; 13(3):42-53. http://tbj.ssu.ac.ir/article-1-1114-en. html

[36] Alizadeh M, KHoshbin S, KHavarpoor F. [Qua;ity of life, wellbeing and depression of Iranian elderly people living in Sydney, Australia (Persian)]. Iranian Journanl of Ageing. 2009; 4(14):15-26. http://salmandj.uswr.ac.ir/article-1-301-fa.html 
This Page Intentionally Left Blank 